\title{
Venous return in acute ischaemic stroke patients measured during computed tomography angiography of head and neck
}

\author{
Bartłomiej Łasocha ${ }^{1}$, Anna M. Grochowska ${ }^{2}$, Paweł Wrona ${ }^{3}$, Paweł J. Brzegowy ${ }^{3}$, \\ Roman Pułyk ${ }^{3}$, Agnieszka Słowik ${ }^{3}$, Paweł R. Latacz ${ }^{3}$, Tadeusz J. Popiela ${ }^{2}$ \\ ${ }^{1}$ Diagnostic Imaging Unit, University Hospital, Krakow, Poland \\ ${ }^{2}$ Chair of Radiology, Jagiellonian University Medical College, Krakow, Poland \\ ${ }^{3}$ Chair of Neurology, Jagiellonian University, Medical College, Krakow, Poland
}

\begin{abstract}
Introduction: The aim of this study was to analyse the general features and usefulness of the time elapsed between the start of contrast agent infusion and its appearance in the aortic arch in acute ischaemic stroke patients subjected to baseline computed tomographic angiography. This is, to the best of our knowledge, the first study of this parameter in a clinical context. We will refer to it hereafter as 'needle-to-aorta delay' (NAD).

Material and methods: The following were recorded: the time it took iodinated contrast media to reach the aorta, the site of occlusion, and automatic perfusion assessments of infarct and salvageable tissue volumes. Demographic data such as age and sex, comorbidities, and clinical factors including heart rate, blood pressure, time elapsed from symptom onset, initial stroke severity, and course of disease, were also assessed.

Results: We analysed 252 cases of stroke. NAD correlated with tissue at risk volume, and was greater for patients with hypertension and atrial fibrillation. The observed time was significantly shorter with less favourable core-to-penumbra ratios. No link was found between NAD and either the rate of infarct progression or the long-term clinical result.

Conclusions: Although no clinical benefit was proven as a result of measuring the time it took contrast media to reach the aorta, our study implies that not only is the brain subject to circulation, but it may also affect its functioning.

Key words: stroke, brain perfusion, penumbra, cardiovascular system

(Neurol Neurochir Pol 2021; 55 (5): 462-468)
\end{abstract}

\section{Introduction}

The effectiveness of mechanical thrombectomy in patients suffering from acute ischaemic stroke is limited to a subgroup of patients which, unfortunately, has not yet been defined in detail. Initially the time-to-window approach was adopted [1]; however, this was undermined in later trials [2-4].

Among the multiple aspects that must be taken into consideration, the progression rate of ischaemia is particularly interesting, because it varies considerably between cases, and interacts closely with potential benefits and time constraints [5]. In most patients, imaging is performed only once for reasons of time optimisation. Therefore, we establish the progression rate based on a single time point and the assumption that the formation of inadvertent lesions begins with symptom onset. Since the availability of automatic volumetric measurements of the infarct core is limited to the highest-level stroke centres, patients outside the therapeutic time window still present a medical and organisational challenge. It is likely that deteriorating circulation impairs brain function [6], and that autoregulation of cerebral bloodflow may be compromised by cardiac output [7]. A connection between blood inflow to the heart and the course of brain ischaemia is to be expected; however, preload measurement is not only cumbersome, but is of dubious value [8].

We investigated the delay between contrast medium injection and its appearance in the aortic arch (we called this

Address for correspondence: Bartłomiej Łasocha, Diagnostic Imaging Unit University Hospital Krakow, Mikołaja Kopernika 36 Str., 31 -501 Krakow,

Poland; e-mail: blasocha@su.krakow.pl

Received: 26.04.2021 Accepted: 22.06.2021 Early publication date: 1.09.2021

This article is available in open access under Creative Common Attribution-Non-Commercial-No Derivatives 4.0 International (CC BY-NC-ND 4.0) license, allowing to download articles and share them with others as long as they credit the authors and the publisher, but without permission to change them in any way or use them commercially. 


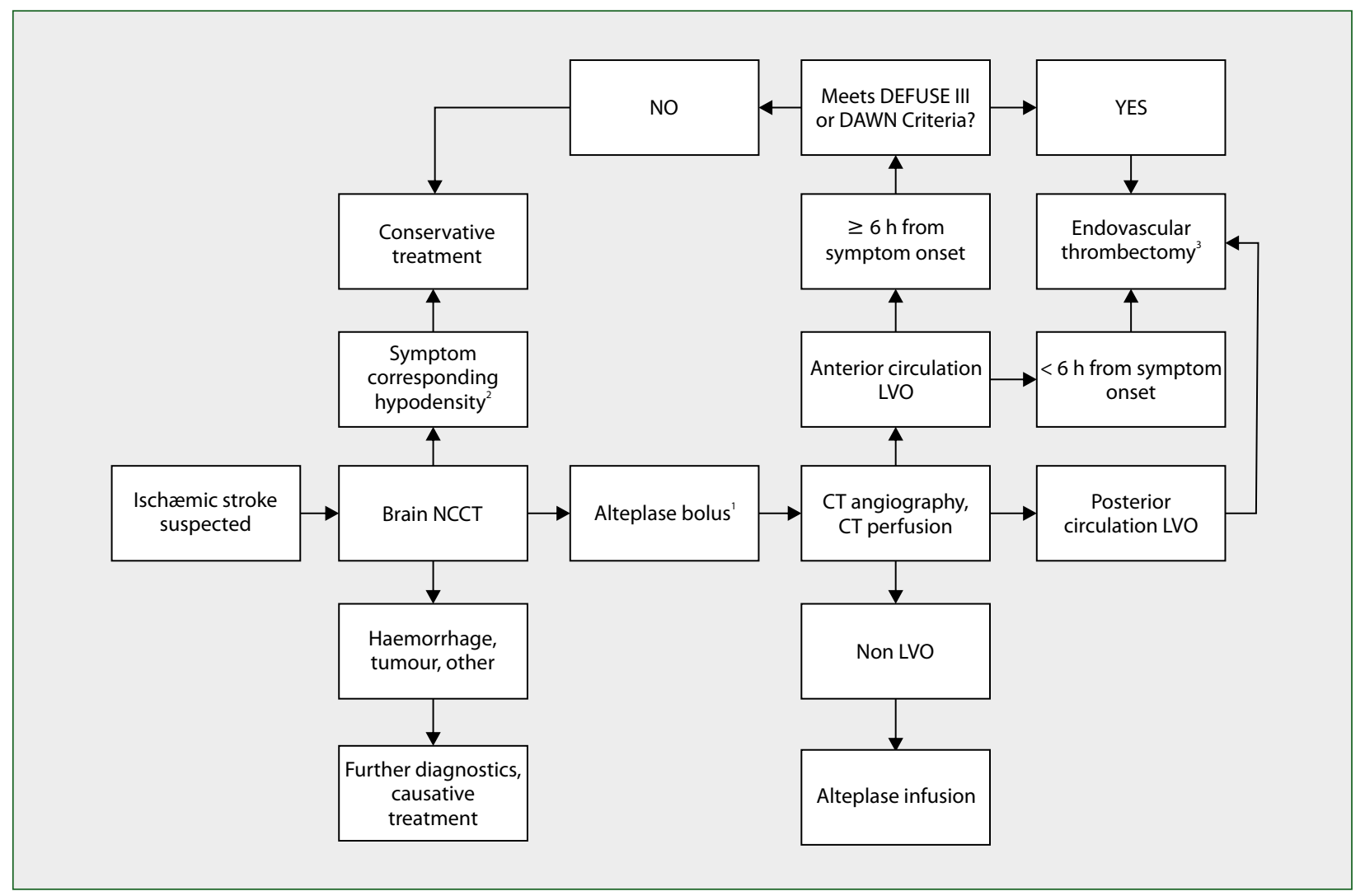

Figure 1. Routine workflow with a patient presenting acute stroke symptoms

'Intravenous fibrinolytic treatment was hedged in by multiple contraindications, the most frequent of which was time from symptom onset exceeding 4.5 hours, and anticoagulation with international normalised ratio (INR) above 1.8. Administration was divided into bolus (10\% of the dose) injected over one minute, followed by pump infusion of the remainder over one hour; ${ }^{2}$ Only regions of hypodensity exceeding one third of middle cerebral artery (MCA) territory, or covering whole area responsible for neurological deficit, were disqualified from causative treatment; ${ }^{3}$ In eligible cases, thrombolytic agent infusion was continued until and during endovascular procedure - the so called 'bridging therapy'

needle-to-aorta delay, NAD) as a potential tool for estimating circulatory function in order to find answers concerning the speed of infarction and to help in qualifying patients for reperfusion treatment.

\section{Material and methods}

We performed a retrospective observational study of patients diagnosed and treated in a comprehensive stroke centre in 2019. A patient flowchart from the emergency department through the imaging unit and on to the neurointerventional lab and stroke department is set out in Figure 1. Medical records and imaging datasets were reviewed to exclude haemorrhage and stroke mimics, to collect information about comorbidities and the course of disease, and to identify radiological features of strokes. The primary aim was to assess NAD as a predictor of outcome; the secondary aim was to assess its linkage with the speed of conversion from penumbra into irreversible damage.
The patient data we analysed comprised the following: age, sex, presence of hypertension, atrial fibrillation, diabetes, initial National Institute of Health Stroke Scale (NIHSS) severity score, eligibility for fibrinolysis, and mechanical thrombectomy. Admission heart rate, arterial blood pressure, serum creatinine level, weight and height were extracted. Echocardiography performed in order to identify cause of stroke provided ejection fraction values. Additionally, in cases of endovascular treatment, reperfusion results were recorded by means of a modified Thrombolysis in Cerebral Infarction (mTICI) score and 90-day follow-up using a modified Rankin Scale (mRS). mTICI grades of $2 b$ and 3 were adopted as standards for successful recanalisation; a good clinical outcome was assumed at mRS scores of 0 and 1 .

Baseline computed tomography (CT) examination consisted of a non-contrast study of brain, head, and neck CT angiography and a brain perfusion CT performed on a 64-slice scanner (Optima CT660, GE Healthcare, Chicago, IL, USA) designated for the use of the emergency department. Intravenous thrombolysis, where applicable, was initiated 


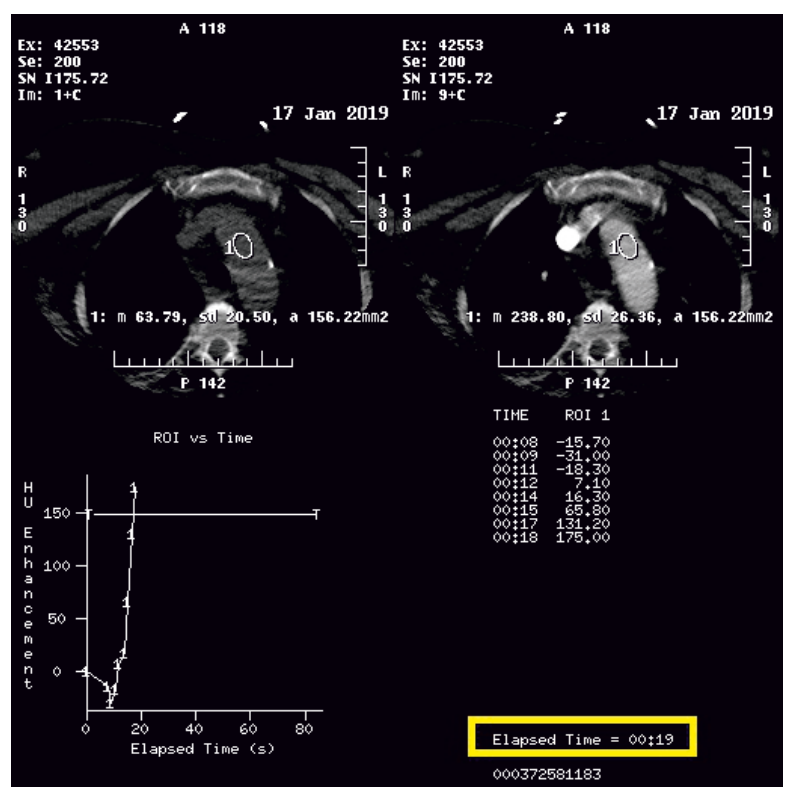

Figure 2. Region of interest (ROI) for bolus triggering, timeenhancement curve, and time delay of scan relative to injection

immediately following the non-contrast study. For vascular and perfusion examinations, a contrast agent containing $350 \mathrm{mg} / \mathrm{mL}$ of iodine (Iohexol) was administered using a dual-head injector in volumes of 80 and $40 \mathrm{~mL}$, respectively. The line was placed in an antecubital vein; an automated bolus triggering technique (SmartPrep, GE Healthcare) was used to optimise opacification of the vessels. NAD was read directly from the SmartPrep report image automatically appended to the study (Fig. 2). The scans were also evaluated for occlusion site and time elapsed from symptom onset to imaging. Volumes of penumbra and infarct core were calculated automatically using rapid processing of perfusion and diffusion (RAPID, iSchemaView, Menlo Park, CA, USA) software, employing thresholds for time-to-maximum (TMAX) over six seconds to delineate tissue at risk, and cerebral blood flow (CBF) below 30 per cent of contralateral brain tissue to discern irreversible damage $[2,3]$. We adopted a mismatch threshold of 1.8 between irreversible damage and tissue-atrisk volumes in order to identify 'penumbral pattern' patients $[3,9]$. Infarct progression rate was calculated as core volume divided by the time elapsed from symptom onset to the first image of the perfusion scan. Based on these measurements, biological indices were then computed, using coefficients published previously [10].

$\mathrm{R}$ software was used for the statistical workup. For categorical variables, frequencies were presented as percentages. Normal distribution of continuous variables was assessed using the Shapiro-Wilk test. Median and interquartile ranges were given for ordinal and numeric variables which did not follow normal distributions. The connections between ordinal and continuous variables were assessed using a Spearman correlation. The Wilcoxon test was used for assessment of
Table 1. Characteristics of study group

\begin{tabular}{lcc} 
Demographic characteristic & $\begin{array}{c}\text { Number } \\
\text { or median }\end{array}$ & $\begin{array}{c}\text { Percentage } \\
\text { or IQR }\end{array}$ \\
\hline Age & 70 & $65-81$ \\
Sex (female) & 131 & $52 \%$ \\
Diagnosed arterial hypertension & 201 & $80 \%$ \\
Admission systolic pressure & 151 & $134-170$ \\
[mmHg] & & \\
Admission diastolic pressure & 85 & $75-95$ \\
[mmHg] & 72 & $29 \%$ \\
Atrial fibrillation & 80 & $71.25-90$ \\
Admission heart rate & 65 & $60-70$ \\
Ejection fraction & 76 & $30 \%$ \\
Diabetes & 26.12 & $24.09-30.42$ \\
Body Mass Index & 79 & $69-96$ \\
Admission creatinine & 10 & $4-17$ \\
NIHSS & 120 & $48 \%$ \\
Fibrinolysis & 74 & $29 \%$ \\
Thrombectomy & & \\
& &
\end{tabular}

categorical factors influencing bolus arrival delay and ischaemic progression rate.

The Bioethical Committee of the Regional Board of Physicians in Krakow waived the need for approval for this study as it was a retrospective analysis.

\section{Results}

The study covered 252 strokes in 247 patients: 131 in females and 121 in males. A summary of patient demographics is set out in Table 1 .

The majority of subjects was hypertensive and overweight. One in four of them exhibited obesity and baseline systolic blood pressure exceeding mild hypertension values. Almost half of cases were eligible for intravenous thrombolysis. The data derived from baseline computed tomography is set out in Table 2. No measurement followed a normal distribution.

The delay in contrast medium inflow was prolonged by atrial fibrillation by four seconds on average $(p<0.001)$. Patients diagnosed with arterial hypertension were characterised by NADs prolonged by two seconds ( $p=0.001)$, but the measured values of arterial blood pressure did not correlate with NAD. Contrast arrival time exhibited a link with ejection fraction $(p<0.001, R=0.383)$, and heart rate and serum level of creatinine correlated with NAD only when we excluded atrial fibrillation patients.

Also, patients with higher NIHSS scores tended towards longer bolus arrival times $(R=0.192, p=0.002)$. The measurement results did not depend on sex, age, weight, height, BMI or the presence of diabetes. However, in patients with longer times from symptom onset who suffered from M1 and tandem occlusions, NAD was shortened $(\mathrm{R}=-0.616, \mathrm{p}=0.002)$. 
Table 2. Imaging findings

\begin{tabular}{|c|c|c|c|}
\hline \multicolumn{2}{|l|}{ Morphological } & Number & Percentage \\
\hline \multirow[t]{5}{*}{ Occlusion site } & $\mathrm{ICA}^{1}$ & 23 & 9 \\
\hline & Tandem ${ }^{2}$ & 23 & 9 \\
\hline & $M 1^{3}$ & 55 & 22 \\
\hline & $M 2^{4}$ & 52 & 21 \\
\hline & other & 99 & 39 \\
\hline \multicolumn{2}{|l|}{ Functional } & Median & IQR \\
\hline \multicolumn{2}{|l|}{ NAD (s) } & 19 & $17-24$ \\
\hline \multicolumn{2}{|c|}{ Time from symptom onset to imaging (min) } & 172 & $99.5-268.8$ \\
\hline \multicolumn{2}{|l|}{ Infarct core $[\mathrm{mL}]$} & 22.5 & $10-58.75$ \\
\hline \multirow{4}{*}{$\begin{array}{l}\text { Infarct progression } \\
\text { rate }^{5}\end{array}$} & Volume $[\mathrm{mL} / \mathrm{min}]$ & 0.131 & $0.058-0.334$ \\
\hline & Neurons $[\mathrm{mln} / \mathrm{min}]$ & 2.761 & $1.223-7.040$ \\
\hline & Synapses [bln/min] & 20.163 & $8.927-51.410$ \\
\hline & Fibre length [km/min] & 17.34 & $7.68-44.21$ \\
\hline
\end{tabular}

'ICA - internal carotid artery; ${ }^{2}$ TANDEM — internal carotid artery and middle cerebral artery occlusion in entire segment with or without visible blood flow in between (through ophthalmic artery or anterior cerebral artery): ${ }^{3} \mathrm{M} 1$ - segment of middle cerebral artery from origin to bifurcation/trifurcation; ${ }^{4} \mathrm{M} 2$ - segment of middle cerebral artery, also known as insular segment; ${ }^{5}$ The rate of infarct progression was calculated only in cases presenting a measurable core (exactly 100 strokes)

Our expected intermediate indicator, i.e. the rate of infarct core growth, remained immutable with regard to age, sex, morphometric parameters, or presence of diabetes, atrial fibrillation, or kidney function impairment. It exhibited a difference of only $0.02 \mathrm{~mL} / \mathrm{min}(\mathrm{p}=0.049)$ between hypertensive (higher) and non-hypertensive patients, and a moderate correlation with measured diastolic blood pressure $(\mathrm{p}=0.009$, $\mathrm{R}=-0.164)$. Faster progression correlated with higher NIHSS scores $(\mathrm{R}=0.543, \mathrm{p}<0.001)$.

No connection to long-term clinical results was found for NAD. Good clinical outcomes remained independent of contrast inflow velocity in every subgroup based on occlusion site, reperfusion results, or age.

In cases where successful recanalisation was achieved, the rate of infarct progression was unrelated to good clinical outcomes $(p=0.088)$.

No connection was observed between NAD and the calculated progression rate, either globally or for subgroups limited to anterior circulation strokes or particular occlusion sites $(\mathrm{p}>0.31)$.

When analysed in relation to neuroimaging, contrast delay did not correlate with the extent of necrosis either in patients overall or in any of the occlusion site subgroups ( $p>0.508$ ).

The time that contrast medium took to reach the aortic arch, however, was linked to the volume of tissue at risk $(\mathrm{R}=0.24, \mathrm{p}<0.001$, Fig. 3$)$, as well as to the proportion of tissue at risk to irreversible injury volumes $(R=0.21$, $p<0.001)$. Surprisingly, the longer it took contrast media to reach the aorta, the greater the mismatch. Unfortunately, the area under the ROC curve plotted for 'penumbral pattern' was only 0.579 (Supplementary Fig. 1).

A strong correlation was found between infarction volume and the rate of infarction. The connection between the extent of ischaemic tissue in baseline examinations and the time elapsed

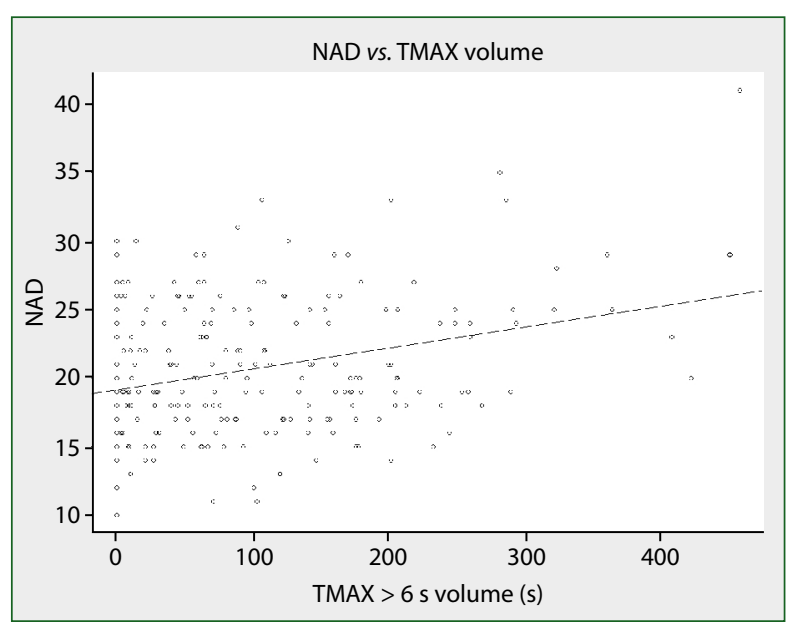

Figure 3. Relationship between needle-to-aorta delay (NAD) and volume of tissue exhibiting time-to-maximum (TMAX) values of 6 seconds or more

from symptom onset was not statistically significant except in the cases of M2 occlusions and core of infarction ( $p=0.50$, $\mathrm{R}=0.237$ ). In all but ICA occlusions, the rate of infarction was linked to the proportions of core and penumbra $(\mathrm{p}<0.001)$.

\section{Discussion}

We expected that slower circulation of blood would promote infarction; however, NAD did not correlate either with the absolute extent of ischaemic lesions nor with their rate of progression. On the other hand, it was closely correlated with the volume of penumbra, something which we had not anticipated. We had presumed the extent of tissue at risk would be determined by the occlusion site, while underestimating global circulatory function. One reason for the above lack of 
correlation may be that more than half of the patients had no inadvertent ischaemic lesions, but only tissue at risk. In the remaining half however, it appears that the smaller the amount of salvageable tissue remaining, the greater the acceleration in circulation.

A reverse cause and effect model might explain this last observation: namely, that cardiovascular functions are influenced by the central nervous system, rather than simply that the brain is subject to circulatory variability. There are numerous pathways along which this mechanism might be executed [11], and, although systemic mobilisation is evident, an intracranial obstacle in tandem with local autoregulation failure eventually leads to cell death [12]. A reduction in NAD in patients experiencing a longer period elapsed from symptom onset suggests that the relationship involves slow, maybe humoral-mediated or otherwise complex, processes rather than a neural reflex. Alternatively, the reflex effect may be cumulative.

We are not certain why NAD failed to correlate with either the extent of necrosis or its progression rate, but, in light of the mechanism proposed above, a negative feedback loop may be assumed: initially sluggish circulation accelerates infarction, which in turn hastens bloodflow. Thus, we may see slow bloodstreams in two totally different populations: rapidly progressing patients who have not yet developed a significant proportion of irreversible damage, as well as patients who, thanks to sufficient (i.e. collateral) blood supply, are protected from inadvertent ischaemia and accelerated circulation. At the same time, an accelerated bloodstream may be present in desperate cases due to agitation, as well as in cases where, thanks to very efficient circulation, patients are protected from the conversion of penumbra into infarct core.

This phenomenon may also negate the ability to predict response to treatment, since $\mathrm{NAD}$, the cardiovascular function marker we acquire, is a combination of a true baseline and the result of the influence of brain ischaemia. We are always presented with these coupled components, rather than with a parameter indicating the initial state of the cardiovascular system.

Delay in contrast arrival may remain intact in minor vessel and isolated ICA occlusions due to compensation by collaterals and weaker disturbances of autoregulatory mechanisms.

Another unforeseen phenomenon involves the correlation of NAD and cardiac ejection fraction. Echocardiography requires an experienced, skilled specialist and a cooperative patient, while modern $\mathrm{CT}$ is already available in most emergency departments, and will become so in even more over time. Although the link between NAD and ejection fraction appears to be very promising, we need to interpret it cautiously. In our study, cardiac sonography was performed to exclude cardiac origin of embolic material, and optimise secondary prevention. Thus, it was scheduled later during hospital stay: on average on the seventh day after symptom onset. Although mathematically the results are bonded tightly, these patients were already after the stroke acute phase and their circulation had already adapted several times: to bed rest, to the initiation of medication, to undergoing procedures, and to physiotherapy.

Measurements performed simultaneously with NAD, or chronic states, also affected contrast media circulation, necessitating an explanation. If increasing peripheral resistance (an inherent component of hypertension) was to blame for slower bolus arrival - either through pure Poiseuille's law or increased cardiac afterload - a correlation with either systolic or diastolic blood pressure is to be expected. Lacking this correlation, but observing delayed inflow in patients diagnosed with hypertension suggests that impaired autonomous circulatory reflexes might be the cause, either by the disease itself or by the initiation of medication. Atrial fibrillation, on the other hand, disturbs a wider spectrum of parameters, impairing left ventricle filling, cardiac rhythm and cardiac cycle alignment, and each of these might render circulation less efficient. An additional burden is the effect of beta-blockers and calcium channel antagonists used in the treatment of this condition.

We must comment as well on the variability of infarct progression rates in our study population. The relationship between velocity and extent of infarction appears trivial, since this ratio is calculated using the former as the quotient and the latter as the numerator. Infarction extent, according to our understanding of ischaemia [13-15] is, however, also proportional to time, used here as the denominator, and so it should nullify the correlation. It happened that the correlation between core volume and time was apparent for M2 occlusions only. This shows the role of collateral vessels $[16,17]$ and calls into question the classic viewpoint of anatomically-based classification of occlusions. Since the neurological deficit is caused by hypoperfused, and not necessarily inadvertently injured, tissue, this plays a role in benefit and risk balancing. Not only the branching pattern, but also the criteria according to which the borders between segments are determined, may be involved in explaining why we fail to address the disease appropriately [18].

An optimistic remark is that successful reperfusion can nullify the effect of infarct progression rates on long term clinical results. We colligate it with improvement of fast progressor prognosis [19]. Analysed patients in this group are fortunate in two different ways: firstly, they were diagnosed early enough so as to maximise the amount of salvageable tissue allowed for endovascular treatment; and secondly, the treatment itself was effective.

It must be stressed that more than half of the analysed patients had no infarcted tissue at the time of the CT, which may affect the results. Moreover, unlike anatomically-based cardiovascular measurements [20-23], NAD uses time to assess function. The same time value serves as a basis for the calculation of the perfusion parameters used to delineate penumbra (TMAX) and infarct core (CBF) $[15,24]$; thus, they may be not as independent as we assume them to be.

Our study contains other flaws: the number of cases is modest considering the inclusion of posterior and small vessel 
strokes. Collected data enabled non-parametric testing only, and clinical follow-up was available only for endovascular cases. However, the parameters and measurements we analysed are either automatic or very well established, rendering them immune to subjectivity and ambiguity.

\section{Conclusions}

The most important finding of our study concerns the influence of global circulatory function on tissue at risk. This poses a challenge to anatomically-based occlusion-site grading of the severity of a stroke.

We also observed an inverse relationship between NAD and the progression of ischaemic lesions. This relationship could not be defined to the point where it might serve as a substitute for mismatch ratio; nor can it be used to detect patients benefiting from reperfusion treatment.

Although routine recording of NAD appears to yield no clinical profit, our study suggests that circulatory function has been underestimated both as a cause of, and as an index of, cerebral hypoperfusion.

Conflict of interest: None. Funding: None.

\section{References}

1. Berkheimer OA, Fransen PSS, Beumer D, et al. A randomized trial of intraarterial treatment for acute ischemic stroke. N Engl J Med. 2015; 1: 11-20, doi: 10.1056/NEJMoa1411587, indexed in Pubmed: 25517348.

2. Jovin TG, Saver JL, Ribo M, et al. Diffusion-weighted imaging or computerized tomography perfusion assessment with clinical mismatch in the triage of wake up and late presenting strokes undergoing neurointervention with Trevo (DAWN) trial methods. Int J Stroke. 2017; 12(6): 641-652, doi: 10.1177/1747493017710341, indexed in Pubmed: 28569123.

3. Albers GW, Lansberg MG, Kemp S, et al. A multicenter randomized controlled trial of endovascular therapy following imaging evaluation for ischemic stroke (DEFUSE 3). Int J Stroke. 2017; 12(8): 896-905, doi: 10.1177/1747493017701147, indexed in Pubmed: 28946832.

4. Thomalla G, Boutitie F, Fiebach JB, et al. WAKE-UP Investigators. Stroke with snknown time of symptom onset: baseline clinical and magnetic resonance imaging data of the first thousand patients in WAKE-UP (Efficacy and safety of MRI-based thrombolysis in wake-up stroke: A randomized, doubleblind, placebo-controlled trial). Stroke. 2017; 48(3): 770-773, doi: 10.1161/STROKEAHA.116.015233, indexed in Pubmed: 28174327.

5. Desai SM, Rocha M, Jovin TG, et al. High variability in neuronal loss. Stroke. 2019; 50(1): 34-37, doi: 10.1161/STROKEAHA.118.023499, indexed in Pubmed: 30566036.

6. Harjola VP, Mullens W, Banaszewski M, et al. Organ dysfunction, injury and failure in acute heart failure: from pathophysiology to diagnosis and management. A review on behalf of the Acute Heart Failure Committee of the Heart Failure Association (HFA) of the European
Society of Cardiology (ESC). Eur J Heart Fail. 2017; 19(7): 821-836, doi: 10.1002/ejhf.872, indexed in Pubmed: 28560717.

7. Liu X, Czosnyka M, Donnelly J, et al. Assessment of cerebral autoregulation indices - a modelling perspective. Sci Rep. 2020; 10(1): 9600, doi: 10.1038/s41598-020-66346-6, indexed in Pubmed: 32541858.

8. Lichtwarck-Aschoff M, Beale R, Pfeiffer U. Central venous pressure, pulmonary artery occlusion pressure, intrathoracic blood volume, and right ventricular end-diastolic volume as indicators of cardiac preload. Journal of Critical Care. 1996; 11(4): 180-188, doi: 10.1016/ s0883-9441(96)90029-5.

9. Campbell BCv, Parsons MW, Campbell BCV, et al. EXTEND-IA Investigators. Endovascular therapy for ischemic stroke with perfusionimaging selection. N Engl J Med. 2015; 372(11): 1009-1018, doi: 10.1056/NEJMoa1414792, indexed in Pubmed: 25671797.

10. Saver JL. Time is brain-quantified. Stroke. 2006; 37(1): 263-266, doi: 10.1161/01.STR.0000196957.55928.ab, indexed in Pubmed: 16339467.

11. Xiao M, Li Q, Feng H, et al. Neural vascular mechanism for the cerebral blood flow autoregulation after hemorrhagic stroke. Neural Plast. 2017; 2017: 5819514, doi: $10.1155 / 2017 / 5819514$, indexed in Pubmed: 29104807.

12. Lo EH, Rosenberg GA. The neurovascular unit in health and disease: introduction. Stroke. 2009; 40(3 Suppl): S2-S3, doi: 10.1161/ STROKEAHA.108.534404, indexed in Pubmed: 19064779.

13. von Kummer R, Dzialowski I. Imaging of cerebral ischemic edema and neuronal death. Neuroradiology. 2017; 59(6): 545-553, doi: 10.1007/s00234-017-1847-6, indexed in Pubmed: 28540400.

14. Agarwal S, Matys T, Marrapu ST, et al. Is CT-based perfusion and collateral imaging sensitive to time since stroke onset? Front Neurol. 2015; 6: 70, doi: 10.3389/fneur.2015.00070, indexed in Pubmed: 25914673.

15. Wintermark M, Flanders AE, Velthuis B, et al. Perfusion-CT assessment of infarct core and penumbra: receiver operating characteristic curve analysis in 130 patients suspected of acute hemispheric stroke. Stroke. 2006; 37(4): 979-985, doi: 10.1161/01.STR.0000209238.61459.39, indexed in Pubmed: 16514093.

16. Vagal A, Menon BK, Foster LD, et al. Association between CT angiogram collaterals and CT perfusion in the interventional management of stroke III trial. Stroke. 2016; 47(2): 535-538, doi: 10.1161/ STROKEAHA.115.011461, indexed in Pubmed: 26658448.

17. Fanou EM, Knight J, Aviv RI, et al. Effect of collaterals on clinical presentation, baseline imaging, complications, and outcome in acute stroke. AJNR Am J Neuroradiol. 2015; 36(12): 2285-2291, doi: 10.3174/ajnr.A4453, indexed in Pubmed: 26471754.

18. Compagne KCJ, van der Sluijs PM, van den Wijngaard IR, et al. MR CLEAN Registry Investigators. Endovascular treatment: The role of dominant caliber M2 segment occlusion in ischemic stroke. Stroke. 2019; 50(2): 419-427, doi: 10.1161/STROKEAHA.118.023117, indexed in Pubmed: 31287757.

19. Rocha M, Jovin TG. Fast versus slow progressors of infarct growth in large vessel occlusion stroke: clinical and research implications. Stroke. 2017; 48(9): 2621-2627, doi: 10.1161/ STROKEAHA.117.017673, indexed in Pubmed: 28794271.

20. Chaturvedi A, Oppenheimer D, Rajiah P, et al. Contrast opacification on thoracic $\mathrm{CT}$ angiography: challenges and solutions. Insights into Imaging. 2016; 8(1): 127-140, doi: 10.1007/s13244-016-0524-3. 
21. Lakoma A, Tuite D, Sheehan J, et al. Measurement of pulmonary circulation parameters using time-resolved MR angiography in patients after Ross procedure. AJR Am J Roentgenol. 2010; 194(4): 912-919, doi: 10.2214/AJR.09.2897, indexed in Pubmed: 20308491.

22. Kathiria NN, Devcic Z, Chen JS, et al. Assessment of left ventricular enlargement at multidetector computed tomography. J Comput Assist Tomogr. 2015; 39(5): 794-796, doi: 10.1097/ RCT.0000000000000279, indexed in Pubmed: 26295194.
23. Sohrabi S, Hope M, Saloner D, et al. Left atrial transverse diameter on computed tomography angiography can accurately diagnose left atrial enlargement in patients with atrial fibrillation. J Thorac Imaging. 2015; 30(3): 214-217, doi: 10.1097/RTI.0000000000000132, indexed in Pubmed: 25629578.

24. Heit JJ, Wintermark M. Perfusion computed tomography for the evaluation of acute ischemic stroke: strengths and pitfalls. Stroke. 2016; 47(4): 1153-1158, doi: 10.1161/STROKEAHA.116.011873, indexed in Pubmed: 26965849. 